\title{
Enhancing the Performance of Wireless Sensor Networks thorugh Clustering and Joint Routing with Mobile Sink
}

\author{
Sujeethadevi T, Bhagyalakshmi L and Sanjay Kumar Suman
}

\begin{abstract}
In wireless sensor network, randomly deployed nodes are formed as a clusters of varying size for each area depending upon the numbers of users. This paper deals with the cluster based joint routing with mobile sink and with static sink in cognitive based wireless sensor network. The Joint Routing (JR) is designed to overcome the problems, due to data gatherings of the sensor nodes for any application. Channel resources usually may vary among the different routing methods based on the traffic characteristics and application they require, which poses a great challenge to guarantee time delivery services. These problems poses a great challenge for cognitive radio based WSN. The resource allocation technique overcomes the problems like spatial priority, time delay, transmission delay and energy loss and here the channel resources are allocated with the help of TDMA technique. The static sink in networks consumes more energy which results the early die out of the nodes. Hence throughput of the networks declines which badly affect the network life time. To overcome these issues, static sink is replaced by mobile sink, which consumes less energy, before each transmission in a sensor networks. The networks with mobile sink provide us optimal solution and performance as well, while comparing with network with static sink. It is shown that the proposed system achieves 15\% of improved throughput, 20\% of less packet loss and 35\% of less delay when compare with the system having centralized sink.
\end{abstract}

Index Terms-Joint Routing, Cluster, mobile sink, TDMA, Static sink, Throughput, Packet Loss.

\section{INTRODUCTION}

Wireless sensor networks (WSN), is no longer the topic of study but they are part of daily life. These networks contains spatially deployed autonomous sensor nodes to sense, measure and control many variable parameters like temperature, sound, pressure, etc. which may vary according to the physical or environmental condition. These sensor nodes are transceiver which collect the information about parameters and cooperatively pass their data through the networks to destination or required locations. The term autonomous sensor defines the capacity of a node as a transceiver, transducer and microcomputer having individual

Revised Manuscript Received on October 30, 2019.

* Correspondence Author

T. Sujeethadevi*, Tata Consultancy Services, Chennai, India.

Dr. L. Bhagyalakshmi, Department of ECE, Rajalakshmi Engineering College, Chennai, India.

Dr. Sanjay Kumar Suman, Department of ECE, Malineni Lakshmaiah Women's Engineering College, Guntur, AP, India.

(C) The Authors. Published by Blue Eyes Intelligence Engineering and Sciences Publication (BEIESP). This is an open access article under the CC BY-NC-ND license (http://creativecommons.org/licenses/by-nc-nd/4.0/) power source. Physically each node is tiny, light weight and movable. Since the sensors work as transducer also, thereby,

WSN is sometimes called wireless sensor and actuator networks (WSAN) [1]. The when a sensor senses some parameter, the sensed parameter is converted into corresponding electrical signal by the transducer in WSN. The sensed data are processed and stored by microcomputer and finally the transceiver part of the networks transmits data to the central computer or receives data from the central computer. To perform these task each node needs power which is supplied by battery embedded with each node. Thus, a sensor node, also known as a mote is capable of sensing and then collecting data, processing and storing data and communicating the processed data in the form of information. It can be noted that all motes are nodes but reverse is not always true [2]. It is also defined as application oriented networks, since depend on the specific applications, these networks might be equipped with GPS device for locating position, a power source and a mobility.

The major challenges in WSN are whether the area is in organized manner or unorganized manner. Clustering primarily focuses on the communication of data process and overcomes the energy related problems which is considered as an efficient methodology to improve the throughput of the system with in-built features that differentiate these networks from other wireless networks such as MANET or mobile cellular networks [3]. Clustering also plays an important role in increasing networks lifetime. Therefore it is a challenging task to select cluster head and the transmission and determination of sink whether to be static or mobile [4][18].

Application of wireless sensor networks is in the entire field either in domestic or in defense. It is equally useful in academic and environmental research \& analysis, communication, home security and in disaster management.It is an growing technology which adds sensing, computation and communication into a single device.

\section{RELATED WORKS}

The review of various papers discussed for our proposal and survey are hinted under this section as follows: Lifetime of the networks can be improved by routing towards a mobile sink. Jun Luo et. al., [5] showed that by using mobile sinks the networks lifetime can be improved. They proposed and simulate Mobi Route protocol for supporting sink mobility. They proved the viability of the mobile sink for expanding lifetime of the networks by using different distribution setups. They assumed controllable and predictable mobility of the nods using the simulator with longer pause time. 
Jin Wang et.al [6] has taken multiple mobile sink nodes for networks where the sink nodes usually moved with a fixed speed to collect sensed data along the network boundary. The influence of multiple numbers of mobile sink nodes was noticeable on the energy consumption and network lifetime. In their research, they addressed impact of the choice of mobile sink node, number and the positions on the performance metrics.

The mesh network becomes very complicated if any failure occurs in that network. Cognitive based [7] networks helps in the above problem by providing the numerical solutions like Lagrangian and Queuing analysis along with the joint routing. Usually joint routing will helps to route one or more data at a time which will improve the energy saving of each nodes and in turn improve the lifespan of the networks. It can also accommodate the traffic and resources availability depending upon its applications. This cognitive based mesh network along with the joint routing uses TDMA for improving the performance gain of the network. But the numerical solutions have some complexity which won't be understandable to ordinary person. Hot Spot problem can be minimized by using Unequal Cluster-based Routing (UCR) protocol. UCR form a group of nodes into cluster of uneven size. The size of the cluster depends on the position of the cluster to save some energy. Normally smaller cluster was deployed near the base station area and the larger cluster was deployed far from the base station.

Amr A. et. al., [8] dealt with joint design of routing and resource allocation algorithms in cognitive radio based wireless mesh networks. In the mesh networks, the spectrum is shared by nodes and the primary users and thus utilizes the overlay model. Before starting the transmission, mesh nodes check the accessible spectrum and channel by sensing the medium like CSMA concept. The system is characterized by queuing theory where, the routing and resource allocation issue is solved as a non-linear integer programming problem.

Yi han et. al., proposed a Joint Channel Assignment and Routing Protocol for Cognitive Radio Wireless Sensor Networks [9]. They explained that the multi-hop transmissions in a single channel restrict the throughput of conservative multi-hop wireless sensor networks because of sharing the resources among all the nodes in the networks. Networks performed well by using multiple channels. However, there is an issue of channel assignment in multi-channel wireless sensor networks that is done carefully while keeping in mind to maximize the performance.

The concepts discussed in this paper and in the following section are cluster based joint routing with static sink and cluster based joint routing with mobile sink. Each cluster head is provided with cognitive sense. Selection of Cluster Head is on the basis of high energy level.

This paper is organized as follows: the concept of clustering technique is presented in section III and the problem is formulated in section IV. Section V and section VI contain the proposed work of clustering technique and joint routing with mobile sink and the simulated results respectively followed by conclusion and references.

\section{CLUSTERING TECHNIQUE}

Clustering is a method of grouping the sensor nodes with respect to certain pattern, it consists of cluster heads. Selection of cluster head is based on rotation scheme such that every node will get opportunity of becoming cluster head. In clustering hierarchical based cluster routing has value for sensor network applications. a large number of sensors are positioned for numerous sensing purposes. If each sensor starts to transmit data, there may be chance of network congestion and hence it will lead to collision of data. Data collision drains the energy which is fixed in the form of battery [2].

Each cluster contains number of sensing nodes known as secondary nodes are led by a head which is cluster head $(\mathrm{CH})$. Clustering forms to two-tier hierarchy in the networks: higher and lower tier formed by $\mathrm{CH}$ and $\mathrm{SN}$ respectively. The role of SNs is to sense and pass their data to the respective CHs. The CHs collects data from all SNs and transmit them to a central base station (BS) directly or via other CHs. By clustering technique, the limited energy is utilized properly and therefore increase the network lifetime. Selection of cluster head is normally random process which may decrease the probabilities energy improvement of network. This type selection may lead to selection of node with low energy. $\mathrm{CH}$ can be mobile or static depend on their ease of maintenance. A static $\mathrm{CH}$ is easy to handle. Moreover a $\mathrm{CH}$ can also act like a base station. Thus clustering is techniques for not only improving the network lifetime but with many features also such as scalability, collision avoidance, load balancing, fault tolerance and QoS [10][17].

All nodes in a particular area will involve in the competition of selection of Cluster Head among themselves. The node with higher energy is regarded as a Cluster Head. Generally clustering involves two phases namely:

\section{- Setup phase}

- $\quad$ Steady state phase

In first phase of clustering technique, clusters are formed each cluster selects its cluster head, while transmission of the collected data by cluster head to the base station is performed in the second phase of operation.

\section{PROBLEM FORMULATION}

\section{A. Issues due to improper clustering}

The delivery of data in WSN application is an important issue. Clustering approach of data transmission is facing number of challenges in terms of distribution challenges, reliable connectivity, selecting efficient $\mathrm{CH}$, choosing optimum cluster size etc. At most care needed while designing the WSN as compared in designing wired networks. The key parameters such that storage constraint, security, communication, limited energy, networks lifetime, QoS must be considered while framing clustering algorithm [11]. A brief explanation the key parameters are:

(a) Storage: The storage space in sensor node is very small and hence it must be taken into account during design process. To meet the storage constraint, queuing technique may be use.

(b) Communication: The coverage area of the sensor must be as large to cover entire region for reliable communication.

(c) Limited limitation: Sensor networks are battery operated hence they are limited by energy consumption. Therefore clustering technique can be used to reduce the energy consumptions as compared to the direct transmission.

Published By: 
(d) Networks Lifetime: Clustering can reduce the energy consumptions and increase network lifetime by implementing Intra cluster communication and multi hop routing schemes.

(e) QoS: Clustering always focus on energy efficiency but does not pay the attention towards the Quality of Service. Hence it is always required to generate a proper clustering algorithm which incorporates the QoS also.

Apart from the above parameters the following parameters are equal important and must be taken into account while implementing clustering technique:

\section{- SCALABILITy}

- LESS LOAD

- REDUCED ENERGY CONSUMPTION

- Fault Tolerance

- Data Aggregation

- LOAD BALANCing

- COllision AvOIDANCE

- QOS

\section{B. Mesh based cluster with CR}

In this method random clusters are formed with mesh topology. These clusters sizes are need not to be equal since random manner is being used in mesh. In each mesh we will have a one CR node which act as a group head i.e. Cluster Head $(\mathrm{CH})$ for a random cluster. We may also have a one or more forwarder in a mesh based on TDMA (Time Division Multiple Access) technique data transmission will occur and reach the sink which is static, there may be one or two in a mesh depending upon the application it needs. This process are done with the help of Recursive algorithm [12].

Use of static sink was more common in early periods of WSN, in that during setup phase; the major energy consumption is the transmission module of each node. Multi-hop communication sends data from source node to sink node. Therefore the energy consumption of the nodes depends on the transmission distance. The transmission distance can be reduced by deploying multiple static sinks and program in such a way that it routes data to the closest sink. Multiple static sinks has to decide where to deploy the nodes either inside the monitored region so that the relay of data can be balanced amongst the nodes.

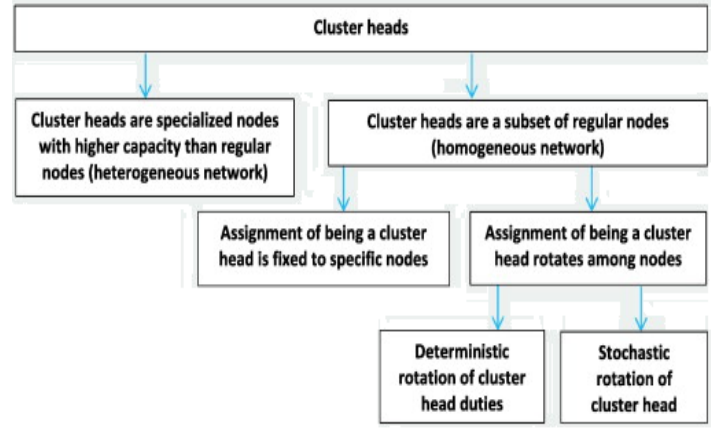

Figure 1. Working of Static nodes

The benefits of multiple static sinks is energy efficiency as shown in figure 1 can also be realized with a single static sink by logically partitioning the sensor field at a two levels either using single level or hierarchically levels. This type of sink can be either static or dynamic, and it can be predetermined or self-organized within the network.

\section{PROPOSED WORK}

The nodes are distributed randomly in the given area say $\mathrm{M} * \mathrm{M}$ and these common nodes are clustered into different circular regions without any common constrain they are termed as clusters. The communication process is done by the common nodes and to their cluster heads and further the cluster heads transmits the data to the mobile sink.

\section{A. System Architecture}

The system architecture is shown as follows:

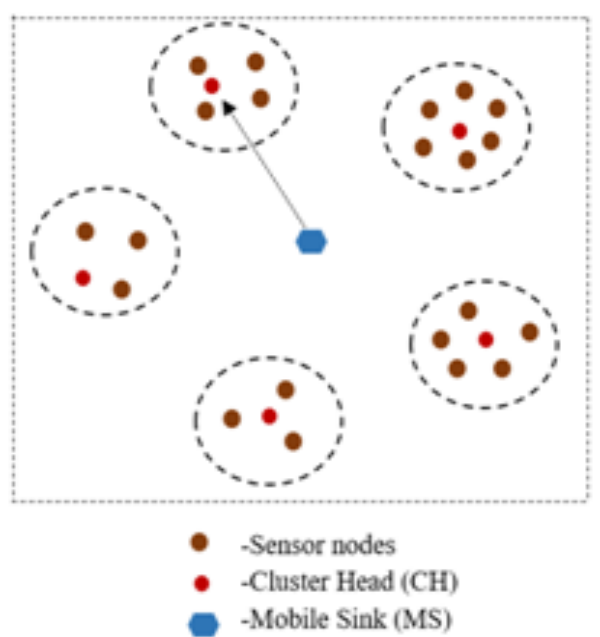

Figure 2. Architecture model

In figure 2 the nodes are distributed in a random manner as an unorganized area with the mobile sink at the center initially. Nodes are arranged in area as random manner which will meet the areas coverage and connectivity. Cluster formed in an area may have equal or unequal number of nodes.

B. Resource Allocation

In this technique the cluster are allocated in a various frequency and within the cluster they communicate among themselves in a slotted time using a TDMA scheme. 


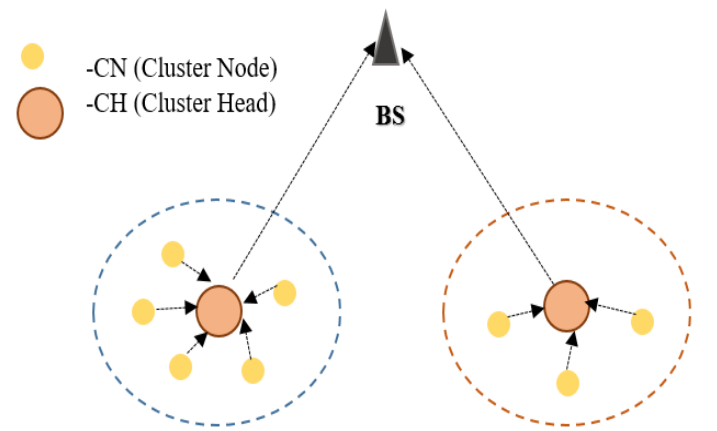

Figure 3. TDMA working

In the above figure shown in figure 3 ,within the cluster all the sensor nodes will involve in a competition to select a clusterhead which is achieved by using a time slots.Time-division multiple access techniques (TDMA) is the one among the multiple access method for shared-medium networks and also defined as channel access method. It permits the users to share the same frequency channel by dividing into different time slots. The cluster heads transmit one after the other, in a rapid transmission by each using its own time slot [12]. This allows multiple cluster heads to share the same transmission medium.

\section{Joint Routing with Mobile sink}

Protocol used for this routing methodology in WSN is DSDV (Destination Sequence Distance Vector).DSDV in sensor networks retains a routing table, which lists of all accessible destinations. For the intermediate destination, the destination node generates a sequence number. The routing table is stored in each node, and then the packets are transmitted between the nodes of a network. Each sensor node of the network streamlines the routing table with a periodical advertisement. The new information is significantly obtainable to maintain the consistency of the routing table with dynamically changing topology of the wireless sensor network [13][14].

To extend the lifetime of the nodes close to the sink, the mobile sink is utilized. To overcome the drawback observed for a static sink, the use of a mobile sink has been proposed. A mobile sink can follow different types of mobility patterns, such as random mobility, fixed path mobility, or controlled mobility, which has some effects with respect to energy efficiency and data collection strategies

Cluster Head $(\mathrm{CH})$ is selected on the basis of high energy in an each cluster. The $\mathrm{CH}$ node is selected among nodes having the highest residual energy and the cluster heads are rotated periodically to balance the energy consumption among the nodes in each cluster. After $\mathrm{CH}$ selection, the communication is done in a 2-tier layer where only $\mathrm{CH}$ is involved in the process of transmission. In the proposed work sink is mobile, it collects the data from particular $\mathrm{CH}$ to a destined area. The mobile sink follows a fixed mobility path to follow a fixed path in a round robin fashion. The fixed path used by mobile sink is predetermined and not influenced by the behavior of the WSN at runtime.

\begin{tabular}{|c|}
\hline \multicolumn{2}{|c|}{ Can the sink predict its future positions? } \\
\hline $\begin{array}{c}\text { If yes, data collector nodes push data to the } \\
\text { sink once it arrives }\end{array}$ \\
\hline
\end{tabular}

Figure 4. Mobile Sink Function
The coverage area of the sensor nodes need to be guaranteed to determine the routing paths for the data packets by using an appropriate strategy. A distinction to be considered as important in this case, is whether the sink can find out its next positions or not. In some cases, sink is able to predict its proposed new position and it can further communicate. It location to a node the sensor node is responsible for collecting the sensed data in its proximity so that when the sink arrives at the sensor node, it is not required to wait for the data [15].

\section{RESULTS}

This section discussed the outputs which are obtained with the help of ns-2 where NS (Version 2) . It is an object oriented, event driven simulator written in $\mathrm{C}++$ and Otcl.The Operating system used will be Linux Ubuntu and as supporting software to use NS 2.34 in Linux Ubuntu 12.04 The Simulation parameter constrains is given in the table 1.

Table 1. Parameters used for model

\begin{tabular}{|l|l|}
\hline Design Parameters & Specification/Value \\
\hline Simulation Software & Network simulator 2. \\
\hline Channel & Wireless \\
\hline Simulation time & $100 \mathrm{Sec}$ \\
\hline Area & $1200 \mathrm{X} 750 \mathrm{~m}$ \\
\hline Speed & $1 \mathrm{~m} / \mathrm{s}$ to $10 \mathrm{~m} / \mathrm{s}$ \\
\hline Routing Protocol & DSDV \\
\hline Propagation model & Two Ray \\
\hline MAC Type & 802.11 \\
\hline Antenna Type & Omni Antenna \\
\hline
\end{tabular}

The parameters discussed here are discussed as follows: And the values obtained here are compared with the system having static and mobile sink

Packet delivery Ratio (Throughput) the loss in packet from the time when an event occurs, to the time when the first packet due to this event is received at the sink shown in figure 5 explains that the throughput observed is good when we compare with the system having static sink

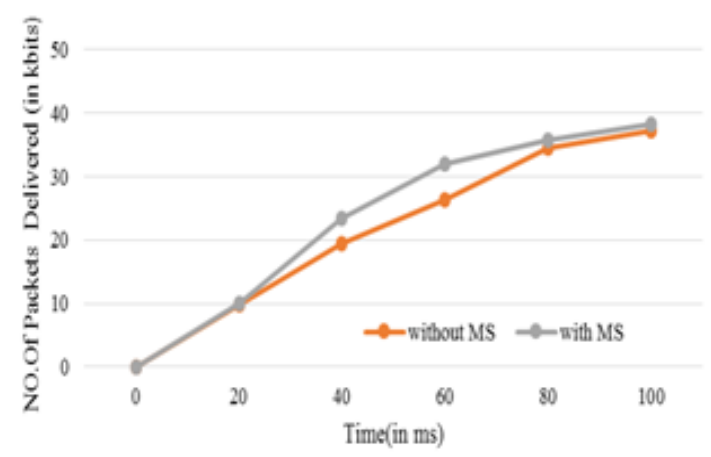

Figure 5. Throughput compared with the system having static and mobile sink

Packet loss shown in figure 6 is defined as the time taken for the packets to reach the network from one endpoint to another.

Published By: 
From the result, we can infer that the packet loss occurred over the time is better in the system with mobile sink which make the system performance better.

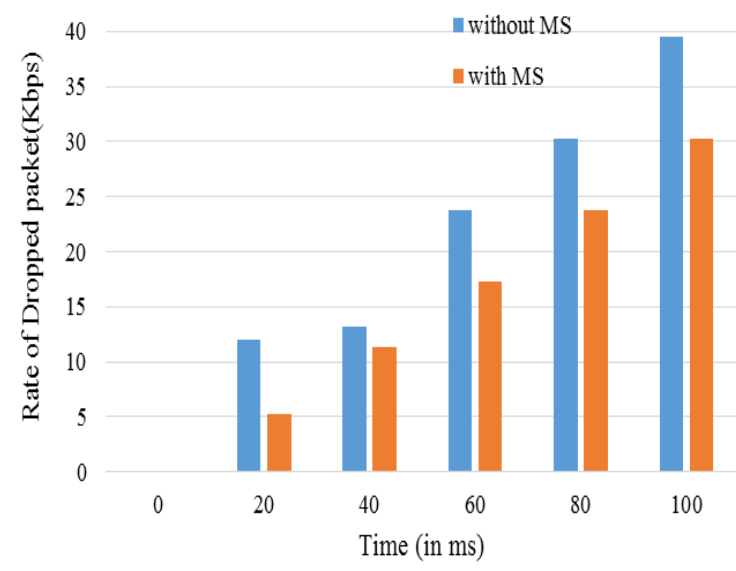

Figure 6. Packet loss compared with system proposed.

End-to-end delay defines as the time taken by a packet to reach the destination after choosing its path. The End to end delay is common term in IP network monitoring and is different from round-trip time (RTT). Delay incurred in figure 7 shows that the less delay being occur in the system with mobile sink [16].

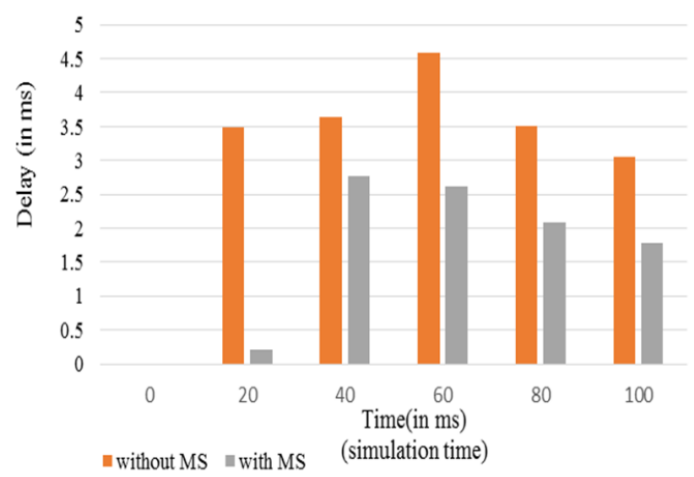

Figure 7. End To End delay of packets

From the above analysis, it has been found that the system having mobile sink will perform in a better manner than the system with the static sink.

\section{CONCLUSION}

This paper proposed a performance improvement in wireless sensor networks by employing combined techniques: clustering and joint routing with mobile sink. Also this work compared the effecto of mobile sink and static sink. Through simulation, the comparison between random mesh cluster with static sink and the proposed mobile sink with performed. The obtained results were analysed and concluded that the networks with mobile sink perform better than that of the networks with static sink.

\section{REFERENCES}

1. Akyildiz, W. Su, Y. Sunknrusubranwniam, and Cayirci .E, "A survey on sensor networks," in IEEE Communication Magazine, Aug. 2002, pp. 102-114.

2. L. Bhagyalakshmi, Sanjay Kumar Suman and K. Murugan, "Corona based clustering with mixed routing and data aggregation to avoid energy hole problem in wireless sensor network", in proceeding of
IEEE International conference on Advance Computing (ICoAC'2012, MIT Chennai), Dec. 2012.

3. Sanjay Kumar Suman, Dhananjay Kumar and L. Bhagyalakshmi, "SINR pricing in non cooperative power control game for wireless ad hoc network", KSII Transactions on Internet and Information Systems, vol. 8, no. 7, pp. 2281-2301, 2014.

4. Sujeetha Devi, Bhagyalakshmi L and Sanjay Kumar Suman, "Cluster based energy efficient joint routing algorithm for delay minimization in wireless sensor networks", International Journal of Pure and Applied Mathematics, vol. 119, no. 15, 307-313, 2018.

5. J. Luo, J. Panchard, M. Piórkowski and J. P.. Hubaux ,"MobiRoute: Routing Towards a Mobile Sink for Improving Lifetime in Sensor Networks", in proceeding of International Conference on Distributed Computing in Sensor Systems, pp 480-497, 2006.

6. Jin Wand, B Li, F Xia, CS Kim and JU Kim, “An Energy Efficient Distance-Aware Routing Algorithm with Multiple Mobile Sinks for Wireless Sensor Networks"Sensors, vol. 14, pp. 15163-15181, 2014.

7. B. Li, H. Li, W. Wang, Z. Hu, and Q. Yin, "Energy-effective relay selection by utilizing spacial diversity for random wireless sensor networks," IEEE Commun. Lett., vol. 17, no. 10, pp. 1972-1975, Oct. 2013.

8. A. A. El-Sherif and A. Mohamed,'Joint Routing and Resource Allocation for Delay Minimization in Cognitive Radio Based Mesh Networks", IEEE Transactions on wireless communications, vol.13, No. 1, pp. 186-197, 2014.

9. Yi-Han $\mathrm{Xu}, \mathrm{Yin} \mathrm{Wu}$ and Jun Song,"Joint Channel Assignment and Routing Protocol for Cognitive Radio Wireless Sensor Networks", Springer Science Business Media New York, 2017.

10. Bhagyalakshmi L and Murugan K, “Avoiding Energy Holes Problem using Load Balancing Approach in Wireless Sensor Network", KSII Transactions on Internet and Information Systems Vol. 8, No. 5, 2014.

11. Sujeetha Devi, Bhagyalakshmi L and Sanjay Kumar Suman, "Cognitive Radio based Resource Allocation and Joint Routing with Various Topologies in WSN", IEEE International Conference on Photonics \& High Speed Optical Networks, ICPHON 18, IEEE Photonic Society, Madras Chapter IEEE, SA Engineering College, April 2018.

12. H. Zhang and P. Gburzynski, "A variable slot length TDMA protocol for personal communication systems," Wireless Personal Communication, vol. 22, no. 3, pp. 409-432, 2002.

13. F. J. Estevez, P. Glosekotter, and J. Gonzalez, "DARAL: A Dynamic and Adaptive Routing Algorithm for Wireless Sensor Networks", Sensors, vol. 16, no. 7, pp. 1-22, 2016.

14. C.F. Shih, W. Liao and H.L. Chao, "Joint routing and spectrum allocation for multi-hop cognitive radio networks with route robustness consideration," IEEE Transaction on Wireless Communication, vol. 10, no. 9, pp. 2940- 2949, 2011.

15. Y. T. Hou, Y. Shi, H.D. Sherali, "Optimal spectrum sharing for multi-hop software defined radio networks," in Proceeding of IEEE International Conference on Computer Communication (INFOCOM), pp. 1-9, 2007.

16. S.K. Kanhere, M. Goudar and V.M. Wadhai, "End-To-End delay optimization in wireless sensor network," International Journal of Smart Sensors and Ad Hoc Networks, vol. 1, no. 3, pp. 2248-9738, 2012.

17. L. Qing, Q. Zhu, and M. Wang, "Design of a distributed energy-efficient clustering algorithm for heterogeneous wireless sensor networks,"Comput. Commun., vol. 29, no. 12, pp. 2230-2237, 2006.

18. C. Fortuna and M. Mohorcic, "Trends in the development of communication networks: Cognitive networks," Elsevier Computer Networks, vol. 53, no. 9, pp. 1354-1376, 2009.

\section{Authors ProfiLE}

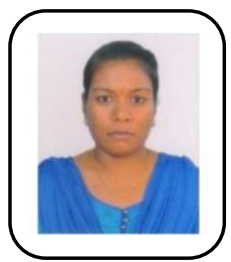

Ms. T. Sujeethadevi received her M.E. in Communication system from Rajalakshmi Engineering College, Chennai, affiliated to Anna University Chennai. Her area of interest in research includes cognitive radio based networks, and she holds a credit of 10 publications comprises journals and conference proceedings. Currently she is working for Tata Consultancy Services, Chennai. Her nature of job is testing using robot framework.

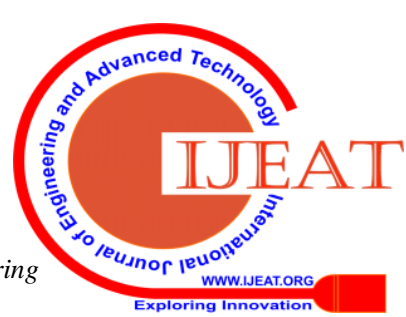




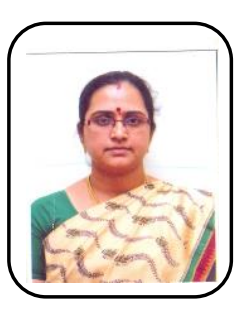

Dr. L. Bhagyalakshmi received her M.E. (Electronics) from Anna University MIT campus, Chennai and Ph.D (ICE) from Anna University, CEG campus, Chennai, India. She holds a credit of more than 50 publications comprises of books, journals and conference proceedings. Her area of interest includes wireless sensor and ad hoc networks, cognitive radio, signal processing, DSP, Microprocessor \& micro-computers, Digital \& Analog Communication and Digital System and Design. Currently she is working as a professor in the dept. of ECE, Rajalakshmi Engineering College, Chennai, India.

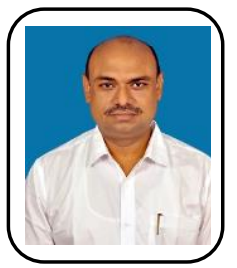

Dr. Sanjay Kumar Suman received his M.E. (ESCE) from NIT Rourkela, India and Ph.D (ICE) from Anna University, MIT campus, Chennai, India. He holds a credit of more than 50 publications comprises of books, journals and conference proceedings. His area of interest includes wireless sensor and ad hoc networks, cognitive radio, signal processing, DSP, Microprocessor \& micro-computers, Digital \& Analog Communication and Digital System and Design. Currently he is working as a Dean of Engineering in Malineni Lakshmaiah Women's Engineering College, Guntur, India. 